\title{
In situ wavelength calibration of the edge CXS spectrometers on $\mathrm{JET}^{\text {a) }}$
}

\author{
E. Delabie ${ }^{1, b)}$, N. Hawkes ${ }^{2}$, T.M. Biewer ${ }^{1}$, M.G. O'Mullane ${ }^{3}$ and JET contributors ${ }^{\text {c) }}$ \\ EUROfusion Consortium, JET, Culham Science Centre, Abingdon, OX14 3DB, UK \\ ${ }^{1}$ Oak Ridge National Laboratory, Oak Ridge, Tennessee 37831-6169, USA \\ ${ }^{2}$ CCFE, Culham Science Centre, Abingdon, Oxon, OX14 3DB, UK \\ ${ }^{3}$ Department of Physics, University of Strathclyde, Glasgow G4 ONG, UK
}

\begin{abstract}
(Presented XXXXX; received XXXXX; accepted XXXXX; published online XXXXX)
A method for obtaining an accurate wavelength calibration over the entire focal plane of the JET edge CXS spectrometers is presented that uses a combination of the fringe pattern created with a Fabry- Pérot etalon and a neon lamp for cross calibration. The accuracy achieved is $0.03 \AA$, which is the same range of uncertainty as when neglecting population effects on the rest wavelength of the CX line. For the edge CXS diagnostic this corresponds to a flow velocity of $4.5 \mathrm{~km} / \mathrm{s}$ in toroidal direction or $1.9 \mathrm{~km} / \mathrm{s}$ in poloidal direction.
\end{abstract}

\section{Introduction}

The measurement of the ion flow speed, using the Doppler shift of an emission line in the visible part of the spectrum, requires a high precision wavelength calibration. This can be particularly challenging when there is no regular access to the front end of the optical fiber bundle to calibrate all tracks on the spectrometer or when standard discharge lamps do not contain enough lines in the spectrum to model the non-linear dispersion accurately enough. In the past, the JET edge charge exchange (CXS) diagnostic ${ }^{1}$, relied on infrequent measurements of the dispersion based on neon or samarium discharge lamps ${ }^{2}$. Variation of the calibration with time was corrected by shifting the wavelength axis under the assumption the emission from a low ionization stage of an intrinsic impurity ( such as $\mathrm{Be}^{1+}$ ) comes from the scrape off layer where the rotation is low. Inter-pulse wavelength calibrations by illuminating a dedicated track on the spectrometer with a neon lamp are now routinely used on $\mathrm{AUG}^{3}$, and a calibration scheme has been developed on DIII-D illuminating the fibers on the tokamak side ${ }^{4}$. The latter is not possible on JET due to space constraints and we present an alternative approach that also enables us to cope with the strongly non-uniform dispersion of the high-throughput, but short focal length spectrometers ${ }^{5}$.

Section II describes the layout of the experimental setup, section III describes the analysis methodology and section IV discusses the resulting wavelength accuracy that is obtained.

\section{Experimental setup}

The layout of the wavelength calibration setup of the JET edge CXS diagnostic is schematically displayed in fig.1. The diagnostic uses two Holospec spectrometers from Kaiser Optical Systems with interchangeable gratings. Before each JET discharge, powerful LEDs $(350-650 \mathrm{~mW})$ that emit light around the wavelengths of interest are switched on for $20 \mathrm{~s}$. The light of the LEDs is collimated and combined using a dichroic beam reflector. The collimated beam of light then passes through a solid Fabry-Pérot etalon. This etalon (thickness $=200 \mu \mathrm{m}$,

${ }^{a)}$ Contributed paper published as part of the Proceedings of the 21st Topical Conference on High-Temperature Plasma Diagnostics (HTPD 2016) in Madison, Wisconsin, USA.

b) Author to whom correspondence should be addressed: delabieeg@ornl.gov.

${ }^{c}$ See the Appendix of F. Romanelli et al., Proceedings of the 25th IAEA Fusion Energy Conference, 2014, Saint-Petersburg, Russia reflectivity $=0.85$, clear aperture $=15 \mathrm{~mm}$ ) is chosen to create a spectral fringe pattern with a free spectral range (FSR) of $0.5 \mathrm{~nm}$, while still transmitting sufficient light. The light is then focused onto a $1 \mathrm{~mm}$ core jumper fiber before being imaged onto a fiber bundle of 4 fibers. The purpose of the jumper fiber is to homogenize the light in the azimuthal direction. If the light would be focused directly onto the fiber bundle, each fiber would collect light from the etalon under a slightly different solid angle, resulting in a slightly different fringe pattern. A fiber from each fiber bundle going towards a periscope of the edge CXS diagnostic is then connected to one of these fibers. A small fraction of the light that has passed through the etalon is backscattered on the optics and the plasma facing window in the periscope. Due to the use of high-power LEDs, only a small fraction of this light needs to be collected along the other fibers in the bundle. After ca. $20 \mathrm{~s}$ of exposure, a well resolved fringe pattern is observed on the spectra from the entire fiber bundle which can be used to calibrate the entire focal plane of the spectrometer. The next problem lies in the cross-calibration of the etalon. For this purpose, one of the fibers connected to the etalon setup is illuminating a dedicated spare track on each of the spectrometers by creating a large spot on a diffuser that is observed from the back. The diffuser enables us to fully fill the etendue of the fibers. The etalon fiber is illuminating the diffuser under a small angle, such that a neon pen-ray calibration lamp can be inserted before the diffuser. This lamp is switched on for ca. $10 \mathrm{~s}$, after the LEDs are switched off, but still before the start of a JET pulse. The combination of having a neon and etalon spectrum on the same track allows to cross-calibrate the etalon (inter-fringe separation and offset).

\section{Methodology}

The analysis of the inter-pulse calibration data consists of 4 steps:

1. The fringe pattern recorded on every track of the camera is fitted with a Gaussian around each peak in the spectrum. This gives a list of pixel positions, $x_{f r}$, corresponding to the positions of the fringes on each of the tracks. Fig. 2 shows an example spectrum with the fit to the fringe pattern.

2. We assume the relation between pixel and wavelength space to be described by a polynomial (eq. (1)). Although analytical expressions for the variation of the dispersion over the focal plane of high resolution transmission gratings 


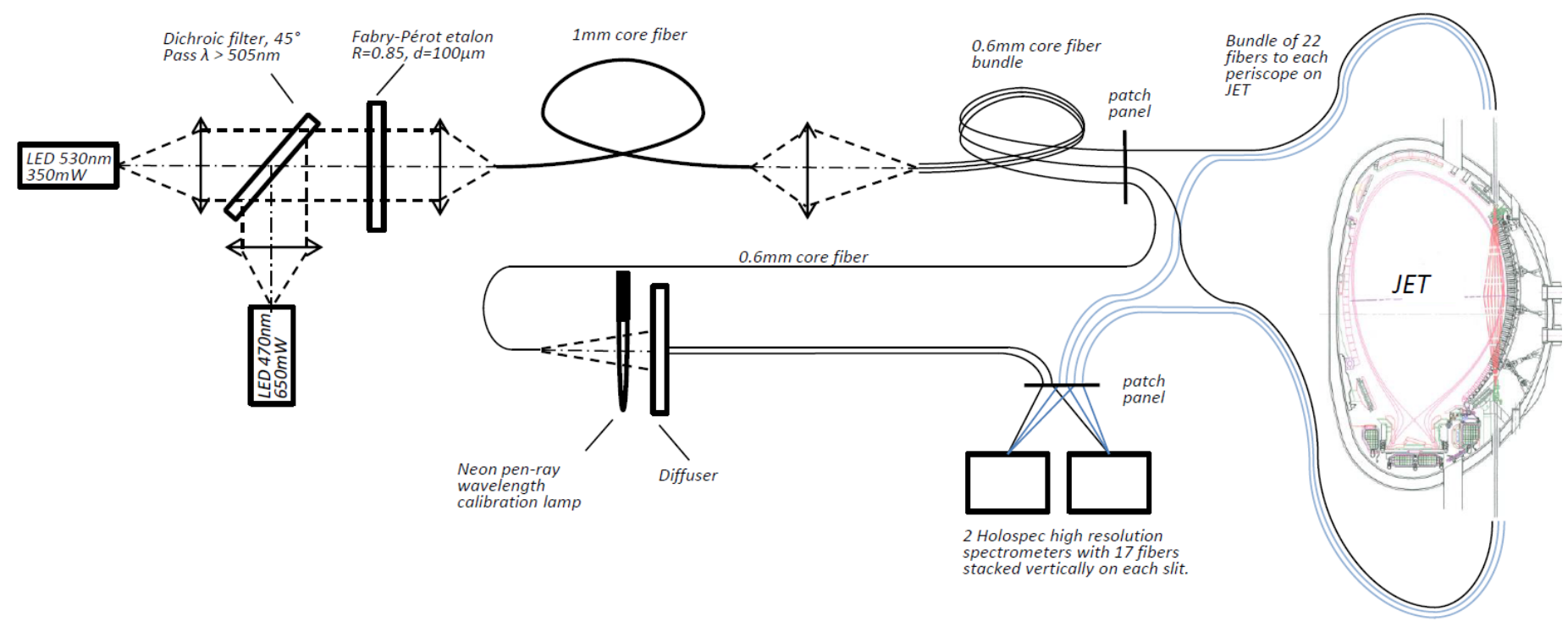

FIG. 1. Schematic layout of the wavelength calibration scheme of the edge charge exchange diagnostic on JET.

as those used for the edge CXS diagnostic have been derived $^{5}$, these expression have many a priori unknown angles that would need to be treated as free parameters when performing a wavelength calibration. Whereas calibrations base on the grating equation have been successfully applied elsewhere, we have taken an alternative approach. We found that a third order polynomial describes the non-linearity of the pixel to wavelength conversion accurate enough to not cause any systematic structure in the residuals from the fit. A parabolic conversion gave a poor fit to both the etalon and neon data.

$$
\lambda=a x^{3}+b x^{2}+c x+d
$$

The distance between two fringes in the etalon pattern is proportional to $\lambda^{2}$. This gives a change in FSR over the spectral range of about $2 \%$, which was found essential to include. Therefore the wavelengths of the fringes, $\lambda_{f r}$, can be parametrized (eq. (2)) depending on just 2 unknowns: $\lambda_{0}$ and $\Delta \lambda_{0}$. We use initial estimates (e.g. from a previous calibration) for the $\lambda^{2}$-dependence of the FSR.

$\lambda_{f r}=\lambda_{0}+\Delta \lambda_{0} C_{f r}$ with: $C_{f r}=\sum_{i=0}^{f r-1} \frac{\lambda_{i}^{2}}{\lambda_{0}^{2}} \approx \sum_{i=0}^{f r-1} \frac{\lambda_{i, i n i t}^{2}}{\lambda_{0, i n i t}^{2}}$

If we substitute eq. (2) in (1), then we find the following function to fit to the fringe positions (eq. (3)).

$$
a^{\prime} x_{f r}^{3}+b^{\prime} x_{f r}^{2}+c^{\prime} x_{f r}+d^{\prime}=C_{f r}
$$

with: $a^{\prime}=\frac{a}{\Delta \lambda_{0}}, b^{\prime}=\frac{b}{\Delta \lambda_{0}}, c^{\prime}=\frac{c}{\Delta \lambda_{0}}, d^{\prime}=\frac{d-\lambda_{0}}{\Delta \lambda_{0}}$

After fitting we obtain a set of coefficients $\left[a^{\prime}, b^{\prime}, c^{\prime}, d^{\prime}\right]$ for each track.

3. We fit a number of well resolved lines from the neon spectrum on the reference track within the spectral region of interest. This results in a list of pixel positions, $x_{i}$, corresponding to a number of well characterized Ne I lines with wavelengths $\lambda_{i}$ from the NIST database ${ }^{6}$. Fig. 3 shows an example neon spectrum from the reference track around the C VI and Ne X CXS lines of interest.

4. In order to obtain $\lambda_{0}$ and $\Delta \lambda_{0}$, the fitted neon positions and coefficients $\left[a^{\prime}, b^{\prime}, c^{\prime}, d^{\prime}\right]$ on the reference track are used. The fit function that is optimized for $\left[\lambda_{0}, \Delta \lambda_{0}\right]$ is given by eq. (4).

$$
a^{\prime} \Delta \lambda_{0} x_{i}^{3}+b^{\prime} \Delta \lambda_{0} x_{i}^{2}+c^{\prime} \Delta \lambda_{0} x_{i}+d^{\prime} \Delta \lambda_{0}+\lambda_{0}=\lambda_{i}
$$

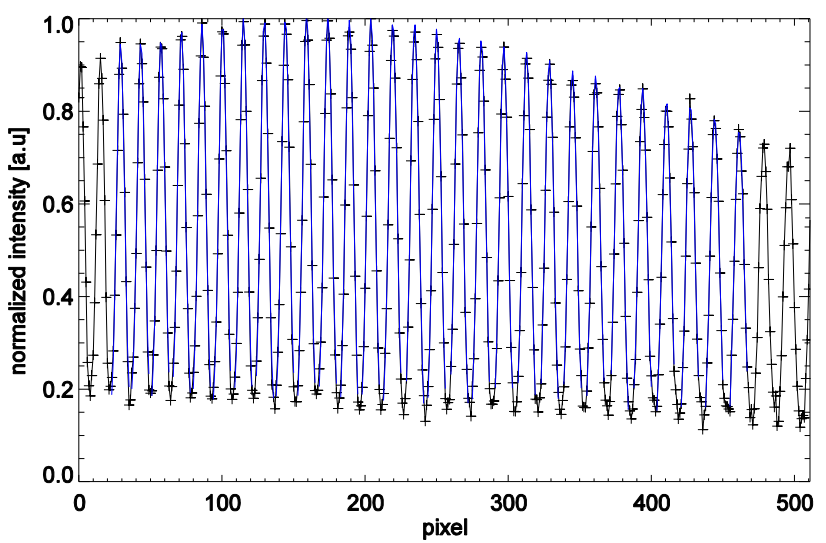

FIG. 2. Fringe pattern obtained by back scattering of light from a LED that has passed through a Fabry-Pérot etalon. The peaks are fitted with Gaussians to extract their pixel positions.

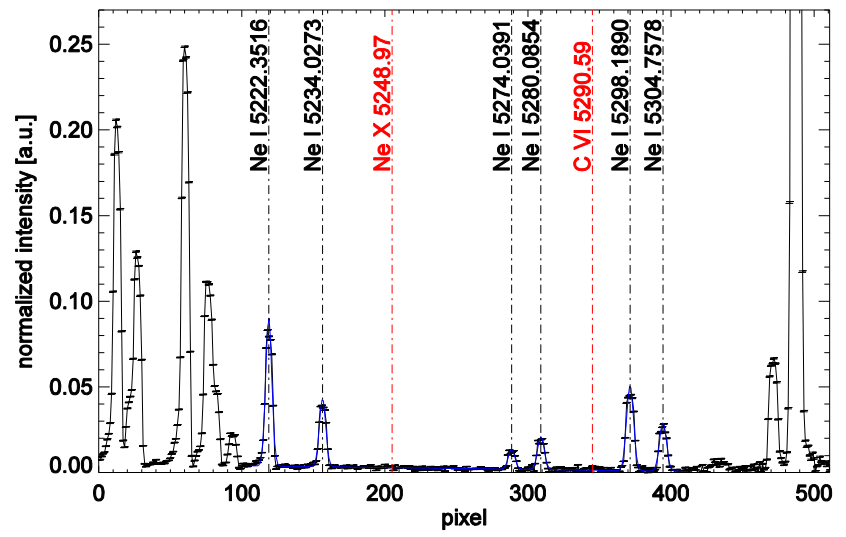

FIG. 3.Fitted neon spectrum on the reference track used to crosscalibrate the etalon. The nominal positions of the C VI and Ne X CX lines are indicated.

With $\left[a^{\prime}, b^{\prime}, c^{\prime}, d^{\prime}\right]$ known for every track and the properties of the etalon $\left[\lambda_{0}, \Delta \lambda_{0}\right]$ defined, we obtain the full pixel to wavelength mapping. 


\section{Discussion of accuracy}

In order to estimate the accuracy of the wavelength calibration, we first look at the misfit with the fitted neon line and etalon fringe positions on the reference track. This is shown in Fig.4(a). There is no obvious structure in the etalon fringe position misfit, which indicates that the $3^{\text {rd }}$ order polynomial that has been used is as good as the data allows. It is worth noting that a $2^{\text {nd }}$ order polynomial resulted in a sinusoidal pattern in the misfit of both the etalon and neon data with amplitude of $0.05 \AA$. The misfit on all neon line positions is within $0.010-0.015 \AA$, this indicates the characterization of the etalon (eq. (2)) is accurate.

In order to come to a better understanding of the wavelength calibration on other than the reference tracks, we have performed a wavelength calibration using the method described above and then immediately connected the fiber normally connected to the reference track to a number of other tracks to measure the difference between the earlier obtained wavelength axis and the neon line and etalon fringe positions. There was no global offset between the reference track and the other tracks, but the misfit was larger than on the reference track by a factor $2-3$. This is illustrated in Fig. 4(b) for a track with a relatively large deviation. It shows the fringe positions from the back reflected etalon light are well fitted, but the difference when the reference fiber is connected can be as large as $0.05 \AA$ on the edge of the spectrum. We believe an explanation lies in the crucial assumption that there is no effect arising from the back scattered light from the etalon filling the fibers on the tokamak side under a potentially different solid angle than during experiments. For future diagnostics this could be eliminated by designing a dedicated reflector on e.g. the shutter of the diagnostic as is being considered for the ITER CXS diagnostics. If we use the variance in the neon misfits as a measure for the obtained wavelength accuracy then we obtain an overall confidence interval of $0.03 \AA$. This corresponds to a line of sight flow velocity of $1.7 \mathrm{~km} / \mathrm{s}$. For the edge CXS diagnostic this translates into either $4.5 \mathrm{~km} / \mathrm{s}$ in toroidal direction or $1.9 \mathrm{~km} / \mathrm{s}$ in poloidal direction.

The obtained accuracy needs to be compared with uncertainties on the natural wavelength of the lines of interest and how this is altered by the selective population of high-1 states in the charge exchange capture process. Cross section effects due to the finite temperature of the receiver come on top of that and can be modelled but are not treated here.

The wavelengths of the Ne I lines used as reference come from NIST ${ }^{6}$ (originally from Burns et al. ${ }^{7}$ ). Comparison with data from previous publications shows a deviation of 0.001-0.002 $\AA$, depending on the line. A later optimization of the energy levels ${ }^{8}$, taking data from various sources, cites an accuracy of $0.0004 \AA$. This is well below the level of concern for this application. Our calculations of the charge exchange wavelengths are in vacuum, but here converted to standard air matching the conversion used in the NIST database (at $529 \mathrm{~nm}, \Delta \lambda=1.4742 \AA$ ). Note that variations in the refractive index of air have no effect on our calibration scheme as this affects the reference lines in the same way as the lines of interest as long as the calculated rest wavelengths are converted to the same standard.

The rest wavelength for the $\mathrm{C}^{5+} \mathrm{n}=8-7$ transition, taking only the Bohr term of the Hamiltonian into account, is $5290.8647 \AA$. If we include spin-orbit coupling and weight the transitions with their populations $\left(p_{i}\right)$ and A-values, the average wavelength $\left(<\lambda>=\sum_{i} p_{i} A_{i} \lambda_{i} / \sum_{i} p_{i} A_{i}\right)$ reduces, largely due to the appearance of satellite lines at lower wavelengths. Because of the change in the population structure, the rest wavelength becomes a function of beam voltage, density and temperature. Fig. 5 shows the average wavelength as function of density for a 25,50 and 75 $\mathrm{keV} / \mathrm{amu}$ neutral beam. The range of variation of the average

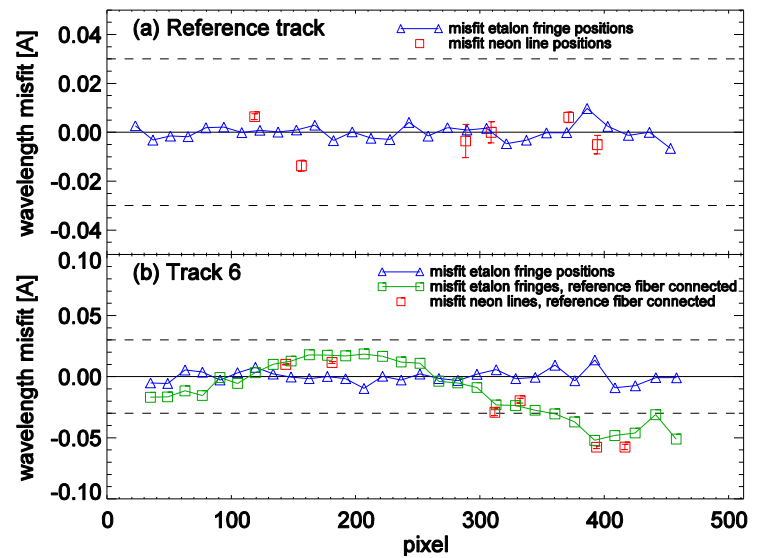

FIG. 4. (a) Difference between the fitted pixel to wavelength conversion and the fitted positions of the neon lines and etalon fringe positions on the reference track of the spectrometer. The fact that there is no structure in the etalon misfit indicates a third order polynomial is as accurate as the data allows. (b) Misfit of the etalon fringe positions on track 6 and difference between the calibrated wavelength axis and neon and etalon fringe positions with the reference fiber connected to track 6 after performing a calibration.

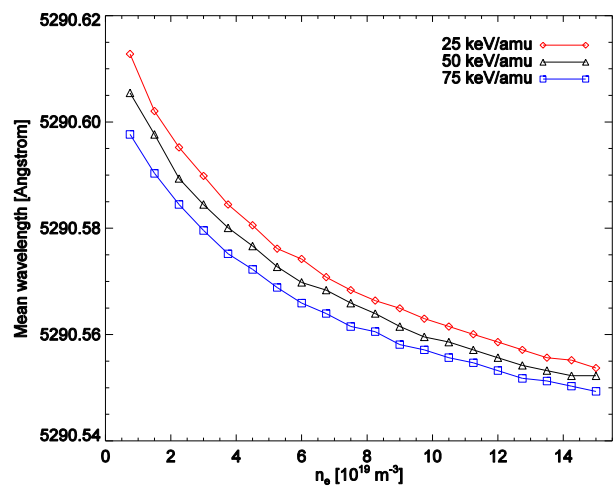

FIG. 5. Variation of the mean rest wavelength of the C VI $n=8-7$ transition following charge exchange capture as function of density for a number of beam voltages. ADAS308 ${ }^{9}$ has been used to obtain the populations and energy levels $\left(\mathrm{T}_{\mathrm{e}}=\mathrm{T}_{\mathrm{i}}=1 \mathrm{keV}\right)$.

wavelength over the typical operational domain of the edge CXS diagnostic $\left(\mathrm{n}_{\mathrm{e}}=1-510^{19} \mathrm{~m}^{-3}\right)$ is about $0.03 \AA$. Hence, we conclude that the uncertainty in the wavelength calibration is similar to the error introduced by assuming a constant rest wavelength.

\section{Acknowledgements}

Work supported, in part, by the US DOE under Contract No. DE-AC05-00OR22725 with UT-Battelle, LLC. This work has been carried out within the framework of the Eurofusion Consortium and have received funding from the Euratom research and training programme 2014-2018 under grant agreement No. 633053.

${ }^{1}$ T.M. Biewer et al., 35th EPS Conf. on. Plasma Physics (2008), Greece.

${ }^{2}$ K. Crombé et al., Rev. Sci. Instrum. 75, No. 10 (2004)

${ }^{3}$ E. Viezzer et al., Rev. Sci. Instrum. 83, 103501 (2012)

${ }^{4}$ P. Gohil et al., Rev. Sci. Instrum. 70, No. 1 (1999)

${ }^{5}$ R. E. Bell et al., Rev. Sci. Instrum. 75, No. 10 (2004)

${ }^{6}$ A. Kramida et al., NIST Atomic Spectra Database (version 5.3),

Available at http://physics.nist.gov/asd (2015)

${ }^{7} \mathrm{~K}$. Burns et al., Journal of the Optical Society of America, 40 (6) p. 339344 (1950)

${ }^{8}$ E.B. Saloman et al., J. Phys. Chem. Ref. Data, Vol. 33, No. 4 (2004)

${ }^{9} \mathrm{H}$. P. Summers, The ADAS User Manual, version 2.6 http://www.adas.ac.uk (2004) 